\title{
Enterprise Group, Cash Holding and Credit Risk
}

\author{
Xiaofeng Xie ${ }^{1,3}$,Yang Yang ${ }^{2}$, Kai X $\mathbf{u}^{1,4}$, Zongfang Zhou ${ }^{1}$ \\ ${ }^{1}$ School of Management and Economics, University of Electronic Science and Technology of China, \\ Chengdu 611731, China \\ ${ }^{2}$ School of Economics Mathematics, Southwestern University of Finance and Economics, Chengdu \\ 611130, China \\ ${ }^{3}$ Genenal Education Department, Chengdu Neusoft University, Chengdu 611844, China \\ ${ }^{4}$ School of tourism and economic management, Chengdu University, Chengdu 610106, China
}

\section{企业集团、现金持有与信用风险}

\author{
谢小风 ${ }^{1,3}$, 杨扬 ${ }^{2}$, 徐凯 ${ }^{1,4}$, 周宗放 ${ }^{1}$ \\ 1 电子科技大学经济与管理学院, 成都 611731 , 中国 \\ 2 西南财经大学金融数学研究所, 成都 611130 , 中国 \\ 3 成都东软学院, 成都, 611844 , 中国 \\ 4 成都大学旅游与经济管理学院, 成都, 610106 , 中国
}

\begin{abstract}
Using the data of Chinese listed companies for 2011-2016 years, on the basis of defining enterprise groups, the relationship between enterprise group, cash holding and credit risk is studied from the perspective of the property property of the final controller of the enterprise group. The research shows that: first, compared with independent companies, the cash holding level of the listed companies belonging to the enterprise group is lower, and the cash holding of the listed companies of the private control group is lower than that of the listed companies of the state-owned control group; secondly, the level of cash holding and the credit wind at the public company level In the end, the negative relationship between cash holdings and credit risk is maintained only in private controlled enterprise groups at the enterprise group level. Besides, this paper also attempts to explore the mechanism behind the relationship between cash holding and credit risk in state-owned enterprises.
\end{abstract}

Keywords: enterprise group; credit risk; cash holding; property right nature

\section{摘要}

利用 2011-2016 年中国上市公司数据, 在 界定企业集团的基础上, 从企业集团最终控制 人产权性质视角研究了企业集团、现金持有与 信用风险之间的关系。研究表明: 首先, 与独 立公司相比, 隶属于企业集团的上市公司现金 持有水平较低, 且民营控制企业集团的上市公 司现金持有比国有控制企业集团的上市公司 现金持有水平更低; 其次, 在上市公司层面的 现金持有水平与信用风险显著负相关, 且国有 控制削弱了这种关系; 最后与传统直观不同, 在企业集团层面, 现金持有与信用风险的负向 关系仅在民营控制的企业集团中被保持。此外， 本文也试图探讨国有控制企业集团现金持有 与信用风险关系背后的机理。

关键词: 企业集团; 信用风险; 现金持有; 产权性 质

\section{1. 引言}

企业集团被定义为通过正式或非正式纽 带相连接, 具有某种共同纲领或目标的独立法 人联合体 ${ }^{[1]}$ 。它是企业发展到高级阶段的一种 组织形式, 广泛地存在于不同的经济体中。已 
有研究表明, 企业集团在各个经济体中都发挥 着举足轻重的作用, 尤其是在新兴经济体中, 企业集团的产业链内部化往往能在一定程度 上弥补市场配置的低效率, 起到了优化经济结 构, 促进经济增长的重要作用 ${ }^{[2]}$ 。我国企业集 团在改革开放以后迅速发展成长壮大, 从 2014 年《财富中国》所公布的中国 500 强企业中可 以看出, 企业集团一共 416 家, 占到企业总数 的 $80 \%$ 以上, 相较 2010 年中国 500 强企业中 企业集团的占比增长了 6 个百分点 ${ }^{[3]}$ 。然而, 要理解企业集团的效率及经济后果却并不容 易。那些具有巨大市场力量的企业集团究竟是 新兴经济体的“天使”, 还是“门口的野蛮人”。 大量的学者提供了不一致的经验证据用以证 明企业集团的效率及经济后果是权变的 ${ }^{[4-6]}$ 。 这背后的原因或许可以视研究视角的不同而 给出不同的解释, 但目前依然缺乏统一的理论 框架。不同的文献甚至在相同的背景下也提供 了矛盾的经验结果 ${ }^{[7]}$ 。关于公司现金持有的理 论分析是一个古老的话题, 许多学者对于一个 公司现金持有的动机、原则、以及影响进行了 研究 ${ }^{[8]}$ 。对于一个独立公司而言, 根据现金持 有的预防性理论, 现金持有可以有效预防外部 环境对该公司的不利影响, 增加公司的财务柔 性, 避免由于现金流问题而导致的财务危机。 因此, 公司较高的现金持有水平能抵抗外界市 场的不利冲击, 有效降低其自身的信用风险。 然而对于企业集团而言, 现金持有水平和信用 风险之间的关系似乎就并非那么直观。这是由 于首先在公司层面上, 企业集团控制下的公司 与独立公司最大的区别在于企业集团可以利 用其内部资本市场进行资源再配置 ${ }^{[9]}$ 。换言之, 对于企业集团控制下的公司而言, 即使自身现 金持有水平较低, 当其遭遇外部不利冲击时, 仍有可能通过内部资本市场高效率调动资金, 进而削弱这些不利冲击对公司的影响。因而对 于企业集团控制下的公司而言, 要维持同样的 信用风险水平, 只需要更低的现金持有水平。 其次产权性质是重要的。一方面, 由于国有资 本背书以及预算软约束 ${ }^{[10]}$, 那些国有控制的企 业集团能够更有效率地为旗下公司提供低成 本资金支持。也就是说对于国有控制企业集团 下的公司而言, 现金持有的增量效应应该较民 营控制企业集团更大, 即国有产权应该在现金 持有与信用风险的关系中表现出显著的正向 调节关系; 而另一方面, 国有产权或许还有“魔
鬼”的一面。已有大量文献强调了由于所有者 主体缺位以及缺乏决策过程不完善, 国有产权 较民营产权更容易出现代理问题 ${ }^{[11]}$ 。而对于现 金持有的研究除了预防性理论, 也有学者提出 现金持有的代理理论。即认为现金是一种相较 于其它资产更容易处置和侵占的资产。管理者 可能通过保持较高的现金持有水平达到侵占 的目的。因此这又为国有产权性质在现金持有 与信用风险关系中的地位提供了一个完全相 反的解释。即国有产权应该在现金持有与信用 风险的关系中表现出显著的负向调节关系。最 后在企业集团的层面, 沿着这样的逻辑若国有 产权总是伴随着管理层代理问题, 而企业集团 由于复杂的内部关系又更容易隐藏管理层侵 占行为 ${ }^{[12]}$ 。所以当国有控制下的企业集团表现 出更严重的代理问题时, 企业集团现金持有与 信用风险之间的关系就值得重估。

因此本文为了系统地回答这几个问题, 首 先利用 2011-2016 的我国沪深 A 股上市公司数 据从最终控制人的角度界定了企业集团; 其次 在控制了公司规模的前提下比较了独立上市 公司与企业集团控制上市公司, 以及国有控制 企业集团下的上市公司与民营控制企业集团 下的上市公司的现金持有水平; 然后考察了隶 属于企业集团的上市公司现金持有与信用风 险之间的关系, 以及产权性质的调节作用; 最 后则在企业集团层面试图探索国有控制企业 集团现金持有与信用风险的关系及背后机理。 除了第一部分引言意外; 第二部分是本文的研 究设计; 第三部分是本文的实证结论及分析; 最后第四部分则是本文的结束语。需要特别说 明的是, 由于受会务组投稿篇幅限制, 本文仅 仅是该主题研究论文的一个精简版概要。更完 整的内容及更细致的研究结论将另文发表。

\section{2. 研究设计}

\section{1 样本及数据}

我们将 2011-2016年我国沪深 A 股上市公 司作为研究的初始样本。根据一下原则对样本 进行清理。删除 (1) 金融行业上市公司; (2) 上市不足一年的公司; (3) 在本文研究区间 内存在数据缺失的上市公司。最终得到有效数 据上市公司 212 家, 包含 1272 个年度-公司研 究样本。为了将隶属于企业集团上市公司与独 立上市公司进行对比。我们对这 212 家上市公 
司按照以下原则进行配对选取 212 家独立上 市公司。(1) 选取配对独立上市公司与样本 公司时间一致; (2) 选取配对独立上市公司 与样本公司行业类型相同或相近; (3) 选取 配对独立上市公司与样本公司资产规模相当;

（4）选取配对独立上市公司与样本公司上市 时间接近。研究数据主要来自于 CSMAR 数据 库。

\section{2 企业集团的界定}

借鉴 Khanna 和 Rivkin $(2001)^{[1]}$, 以及蔡卫 星等 (2015) ${ }^{[13]}$ 的工作, 在本文框架下, 若某 家上市公司的最终控制人在同一年度控制了 两家及以上的上市公司, 这该最终控制人被视 为某个企业集团的控制人, 代表一个企业集团。 同时该上市公司被认为是隶属于该企业集团 的一家上市公司。进一步, 若最终控制人为各 级政府、国务院相关部委及下属机构 (例如财 政部、国资委），则由该控制人所代表的企业 集团被认为是国有产权性质; 否则则被认为是 民营产权性质。在界定企业集团的时候，我们 从上市公司池中采取了随机抽样, 层层追溯的 方式。

\section{3 现金持有的度量}

借鉴 Opler 等(1999) ${ }^{[14]}$ 的相关工作，定义 现金持有如下:

现金持有 $=$ 现金及现金等价物 / (总资产-现金 及现金等价物）

该现金持有的定义也被 Ozkan 和 $\operatorname{Ozkan}(2004)^{[15]}$, 以及蔡卫星等 (2015) ${ }^{[13]}$ 等 文献广泛应用。

\section{4 信用风险的度量}

大量已有文献对信用风险的度量问题展 开了研究。包括 Z-Score ${ }^{[16]}$, Logit ${ }^{[17]}$ 、Probit ${ }^{[18]}$ 、 神经网络 ${ }^{[19]}$ 、粗粮集 ${ }^{[20]}$ 等一系列不同类型的方 法被许多学者提出和改进, 以适应于不同应用 情境下的信用风险度量。由于本文数据来自于 我国沪深 A 股上市公司, 来自证券市场的数 据更能有效反应其信用风险的变化情况。为此 我们选用 KMV 模型来作为信用风险的评价方 法, 用违约概率 EDF 作为本文信用风险的度 量。需要说明的是, 由于我们对企业集团的界 定在于最终控制人的归属, 在企业集团层面探 讨信用风险时需要对企业集团的整体财报进 行并表分析。因此当本文在企业集团层面探讨
时所用的股权价值波动率是经过规模调节后 的企业集团所属各个上市公司股权价值波动 加权。

根据 $\mathrm{KMV}$ 模型设定, 价值变化服从集合 布朗运动。即

$$
V=V_{0} N\left(d_{1}\right)-D P \times e^{-r t} N\left(d_{2}\right)
$$

$$
\text { 其中, } d_{1}=\frac{\ln \left(V_{0} / D P\right)+\left(r+\frac{1}{2} \sigma_{V}^{2}\right) T}{\sigma_{V} \sqrt{T}}, d_{2}=
$$

$d_{1}-\sigma_{V} \sqrt{T,} \sigma_{E}=\frac{V_{0} N\left(d_{1}\right)}{V} \sigma_{V}$ 。

因此, 违约概率为

$$
\mathbf{E D F}=\mathbf{1}-\mathbf{N}(\mathrm{DD})
$$

其中违约距离, $\mathrm{DD}=\frac{\mathrm{v}_{0}-\mathrm{DP}}{\mathrm{v}_{0}} \sigma_{\mathrm{V}}$

\section{5 模型设定}

为了考察本文前述的几个问题, 本文建 立以下几个模型。

$$
\text { Cashingholdings }=\alpha+\alpha \text { Group }+\mathrm{X} \beta+\varepsilon
$$

模型 1 如式(4)所示, 我们关心的是 Group 变量的系数 $\boldsymbol{\alpha}$, 若回归结果显示系数 $\boldsymbol{\alpha}$ 显著为 负, 则意味着隶属于企业集团的上市公司现金 持有水平较独立上市公司现金持有水平更低; 相反若回归结果显示系数 $\boldsymbol{\alpha}$ 显著为正, 则表示 隶属于企业集团的上市公司现金持有水平更 高。在 (4) 中, $\boldsymbol{X}$ 表示本文所选取的控制变 量。参照 Dittmar 等(2003) ${ }^{[21]}$ 等的工作, 控制 变量包括了: 营运资本率 (Wcap)、公司规 模 (Size) 、资产负债率 $(\mathrm{Lev})$ 、资本支出 比率（Capex）、经营现金流（CashFlow）、 股利分配 (Dividend)、股权集中度 (EquFo)、 净增长率 (NetGrowth) 以及应收账款周转率 (Turnover)。

在此基础上我们构建了模型 2 , 在模型 1 的基础上加入了 Group 变量和产权性质变量 (Ownership) 的交互项以分析隶属于国有控 制企业集团与隶属于民营控制企业集团的上 市公司现金持有水平是否有区别。模型 2 如 (5) 所示

$$
\text { Cashingholdings }=\alpha+\alpha \text { Group }+
$$
$\gamma$ Ownership $+\eta$ Ownership $\times$ Group + 
$X \boldsymbol{\beta}+\varepsilon$

(5)

在模型 1 中 Group 变量的系数 $\boldsymbol{\alpha}$ 显著为负 的前提下, 模型 2 中系数 $\boldsymbol{\eta}$ 显著为负, 则说明 相较于那些隶属于民营控制企业集团的上市 公司而言, 隶属于国有控制企业集团的现金持 有水平更低。

进一步, 为验证现金持有水平与信用风险 之间的关系, 我们建立了模型 3 和模型 4 。分 别如 (6) 和 (7) 所示。

$$
\begin{gathered}
\text { CreditRisk }=\alpha+\alpha \text { Cashingholdings }+X \beta \\
+\varepsilon \\
\text { CreditRisk }=\alpha+\alpha \text { Cashingholdings }+ \\
\gamma \text { Ownership }+\eta \text { Ownership } \times \\
\text { Cashingholding }+X \beta+\varepsilon
\end{gathered}
$$

在模型 3 中, 若 Cashingholding 变量的系 数 $\boldsymbol{\alpha}$ 显著为负, 则验证了现金持有预防性理论, 即表明随着现金持有水平的增加信用风险降 低; 若 Cashingholding 变量的系数 $\boldsymbol{\alpha}$ 显著为正, 则说明随着现金持有水平的增加信用风险增 高, 这或许是现金持有代理理论的一个标志。 模型 4 则在模型 3 的基础上加入了现金持有水 平和产权性质变量 (Ownership) 的交互项。 在变量的系数 $\boldsymbol{\alpha}$ 显著为负的前提下, 若模型 4 中变量系数 $\boldsymbol{\eta}$ 显著为正则意味着国有产权性质 削弱了现金持有水平对信用风险的负面影响; 反之若模型 4 中变量系数 $\boldsymbol{\eta}$ 显著为负则意味国 有产权性质加强了现金持有水平对信用风险 的负面影响。

需要说明的是, 模型 1 和模型 2 的回归样 本为全样本 424 家上市公司 (总共 2544 个样 本: 包括 212 家隶属于企业集团的上市公司对
应的 1272 个年度-公司样本和 212 家独立上市 公司对应的 1272 个年度-公司样本）; 模型 3 和模型 4 则分别对隶属于企业集团的 212 家上 市公司的 1272 个年度-公司样本和 212 家独立 上市公司的 1272 个年度-公司样本各自进行回 归。因此模型 3 和模型 4 对两份样本的回归结 果比较将有利于帮助我们解释隶属于企业集 团的上市公司与独立上市公司相比, 其现金持 有与信用风险之间的关系, 以及产权性质对这 种关系的调节。

最后, 再次利用模型 3 试图在企业集团层 面探讨产权性质对现金持有与信用风险之间 关系的影响。为此我们首先将 212 家隶属于企 业集团的上市公司层层追溯, 通过最终控制人, 将其界定为 67 家企业集团。其中国有控制企 业集团占 43 家，占 64\%。民营控制企业集团 24 家, 占 36\%; 然后将每家企业集团报表合 并, 并使用企业集团旗下所有上市公司的现金 持有总和作为企业集团的现金持有水平, 使用 规模调节后的市场波动率作为企业集团的波 动率如前文所述; 最后利用模型 3 分别对 43 家国有控制企业集团的 258 个年度样本和 24 家民营控股企业集团的 144 个年度样本进行 回归, 比较回归结果。

\section{3. 实证结论及分析}

对于本文所使用的面板数据, 在方法上我 们采用了混合最小二乘估计 (pooled OLS) 和 随机效应模型 (random effect, RE) 这两种常 见的估计方式。由于篇幅限制, 在本文中我们 只报告混合最小二乘估计的回归结果, 且不再 列示控制变量的回归结果。更细致的结论将另 文发表。

表 1 显示了混合最小二乘估计下模型 1 和 模型 2 的全样本回归结果。

表 1 企业集团、产权性质和现金持有的回归结果

\begin{tabular}{ccc}
\hline & 模型 1 & 模型 2 \\
\hline Group & $-0.0310^{* * *}$ & -0.0239 \\
Ownership $\times$ Group & & $0.0469^{* *}$ \\
\hline R-Square & 0.4273 & 0.3892 \\
\hline
\end{tabular}

从表 1 的回归结果来看, 模型 1 和模型 2 的预期是一致的。当隶属于企业集团的公司受 都具有较好的解释性。模型 1 中 Group 变量的 到外部市场不利冲击时, 企业集团的内部资本 市场将起到高效配置资源, 缓冲不利冲击的重 系数显著为负 (估计值为 $-0.0310, \boldsymbol{t}$ 值为 -5.72 )。 这表明相对于独立上市公司而言, 隶属于企业 集团的上市公司现金持有水平更低。这和我们 要作用。模型 2 的 Group 变量回归系数为负, 而产权性质与 Group 变量的交叉项系数, 这和 
我们的预期不符。这意味着即便具有国有产权 背书的上市公司在融资成本和便利性上都低 于隶属于民营控制企业集团的上市公司, 但隶 属于国有企业集团的上市公司在现金持有水
平上却更大。

表 2 显示了混合最小二乘估计下模型 3 和 模型 4 在企业集团上市公司和独立上市公司 两类样本下的回归结果。

表 2 信用风险、产权性质和现金持有的回归结果

\begin{tabular}{ccccc}
\hline & $\begin{array}{c}\text { 模型 } 3 \\
\text { (集团样本) }\end{array}$ & $\begin{array}{c}\text { 模型 } 4 \\
\text { (集团样本) }\end{array}$ & $\begin{array}{c}\text { 模型 3 } \\
\text { (非集团样本) }\end{array}$ & $\begin{array}{c}\text { 模型 4 } \\
\text { (非集团样本) }\end{array}$ \\
\hline $\begin{array}{c}\text { Cashingholding } \\
\text { Ownership } \\
\times \text { Cashingholding }\end{array}$ & $-0.0181^{* * *}$ & $-0.0143^{*}$ & $-0.0103^{*}$ & -0.0082 \\
\hline R-Square & & $0.0039^{* * *}$ & & $0.0028^{*}$ \\
\hline & 0.2113 & 0.2141 & 0.3921 & 0.3938 \\
\hline
\end{tabular}

表 2 分别从集团样本和非集团样本的角 较于独立上市公司对降低信用风险的边际贡 度揭示了现金持有与信用风险之间的关系, 以献高大约 $40 \%$; 最后无论是隶属于企业集团的 及产权性质在其中的调节作用。首先从表 2 中 上市公司还是独立上市公司, 国有产权都显著 可知在非集团样本下的现金持有对信用风险削弱了现金持有对信用风险的缓冲作用。对于 的解释力更强。也就是说, 相较于隶属于企业企业集团背景的上市公司而言, 这种削弱更加 集团的上市公司, 独立上市公司的现金持有对明显。 信用风险的影响更具解释力; 其次, 无论是隶 属于企业集团的上市公司还是独立上市公司, 其现金持有水平的提高都显著降低了信用风 险。对于隶属于企业集团的上市公司而言, 现 金持有的回归系数为 -0.0143 , 而对于独立上市 公司而言, 该回归系数为- 0.0103 。也就是说企 业集团背景的上市公司提高现金持有水平相

为尝试检验国有控制削弱现金持有对信 用风险缓冲的原因, 我们进一步细分样本。企 业集团背景上市公司样本按照最终控制人产 权性质又分为国有控制和民营控制。分别利用 模型 3 对其回归, 再此考察不同产权下集团样 本现金持有对信用风险的影响。表 3 向我们展 示了回归结果。

表 3 信用风险、现金持有的集团样本回归结果

\begin{tabular}{ccc}
\hline & $\begin{array}{c}\text { 模型 3 } \\
\text { (国有控制) }\end{array}$ & $\begin{array}{c}\text { 模型 3 } \\
\text { (民营控制) }\end{array}$ \\
\hline Cashingholding & -0.0114 & $-0.0209^{* *}$ \\
\hline 观测值 & 258 & 144 \\
R-Square & 0.1783 & 0.2058 \\
\hline
\end{tabular}

从表 3 的结论中可以看出, 在进一步细化 现金持有的核心目的是为了应对外界不利冲 样本以后, 国有控制的企业集团上市公司其现 金持有对信用风险的影响不再显著。换言之, 现金持有水平的提高缓冲信用风险的结论, 即 现金持有的预防性理论仅在民营控制下得到 了保持。我们有理由相信, 由于企业集团管理 层侵占的隐蔽性, 现金持有的代理理论主导了 企业集团的现金持有水平。当然, 根据表 3 的 回归结果, 这还只是我们的一个推测。如前文 所述, 更细致的讨论我们将另文发表。

\section{4. 结束语}

关于现金持有的动机, 已有文献给出了 多种解释。其中最主流的预防性理论认为公司 击对自身的影响, 避免公司由于现金流紧张而 陷入财务危机。在这样的直观下, 预防性理论 自然地认为现金持有水平的提高可以降低公 司的信用风险。另一方面, 我们也提供了现金 持有的另一个替代性理论, 即代理理论。该理 论认为现金或现金等价物较其它资产更容易 被侵占, 因此较高的现金持有水平或许暗示了 严重的代理问题。换言之, 该理论预测现金持 有水平的提高并不能降低信用风险, 甚至可能 由于严重的侵占行为提高公司信用风险。有文 献并没有提供相关经验证据。那么究竟我国公 司现金持有的根源为何? 已有文献鲜有涉及, 
并尚无相关问题的经验证据。考虑到由于所有 者长期缺位导致的严重的国有产权代理问题, 以及企业集团这种更容易隐藏侵占行为的组 织形式, 本文选取我国上市公司作为样本, 分 析了企业集团背景下现金持有与信用风险的 关系, 以及产权性质对这种关系的影响。

首先, 本文通过隶属于企业集团的上市 公司和独立上市公司样本现金持有水平的对 比发现, 隶属于企业集团的上市公司现金持有 水平更低。这符合我们的预期, 解释了企业集 团内部资本市场作为成员企业风险盾的重要 作用; 同时在加入产权性质作为调节变量后我 们发现国有控制下的集团化对于降低现金持 有水平的贡献较民营控制下的集团化更低。当 然, 这也可能是由于两方面的原因: 第一, 国 有控制企业集团的内部资本市场效率较民营 控制企业集团内部资本市场低; 第二, 国有控 制企业集团较民营控制企业集团具有大量的 融资优势, 因此长期处于 “不缺钱”的状态。其 次, 本文考察了现金持有与信用风险之间的关 系。无论是集团样本还是非集团样本, 现金持 有水平的提高都显著降低了信用风险。这揭示 了现金持有预防性理论的一面。然而国有控制 显著削弱了现金持有对降低信用风险的贡献, 特别是企业集团而言, 这种削弱更为严重。这 可能是由于企业集团代理问题的严重性导致。 为此, 最后我们进一步细化的样本。将集团样 本进一步分为国有控制和民营控制两类, 分别 考察两类样本下现金持有水平对信用风险的 影响。回归结果表明, 仅民营控制的集团样本 为耻了现金持有的预防性理论, 国有控制下的 集团样本更倾向于现金持有的代理理论。因此, 我们有理由相信, 现金持有动机的预防性解释, 亦或代理解释是一个权变的过程。正如前文所 言, 本文受篇幅限制, 仅仅起到“抛砖引玉”的 作用。更细致的研究结论和进一步的讨论我们 正在进行, 将在后续另文发表。

\section{Acknowledgements}

This study was supported by National Natural Science Foundation of China (No. 71271043 \& No.71701066)。

\section{致谢}

本研究得到了国家自然科学基金项目 (71271043、71701066)的资助。

\section{参考文献}

[1] T. Khanna, J. W. Rivkin. Estimating the performance effects of business groups in emerging markets. Strategic. Management Journal, 2001, 22(1): 45-74.

[2] T. Khanna, K. Palepu. The future of business groups in emerging markets: long-run evidence from Chile. Academy of Management Journal, 2000, 43(3):263-285.

[3] 杨扬. 企业集团信用风险传染与演化机 理研究. 成都: 电子科技大学博士学位论 文,2015.

[4] K. Lins, H. Servaes. International evidence on the value of corporate diversification. Journal of Finance, 1999,54(6):2215-2239.

[5] J. He, X. Mao, O. Rui, X. Zha. Business group in China. Journal of Corporate Finance, 2013, 22:166-192.

[6] T. Khanna, Y. Yafeh. Business groups in emerging markets: paragons or parasites? Journal of Economic Literature,2007, 45(2):331-372.

[7] M. Camey, D. Shapiro, Y. Tang. Business group performance in china: ownership and temporal consideration. Management and Organization Review, 2009, 5(2):167-193.

[8] J. M. Keynes. The general theory of employment, interest and money. McMillan, London, 1936.

[9] 杨扬, 周宗放, 肖珢. 企业集团道德风险 与信用风险的作用机理研究.系统工程学 报, 2012, 6(27):806-811.

[10] 徐朝阳. 作为政策工具的国有企业与国 企改革:基于软约束的视角 [J].中国软科 学,2014, 3:32-44.

[11] 张驰. 国企代理成本: 博弯模型的推演、 最优解及分析. 财会研究, 2016, 11:52-54.

[12] K. H. Bae, J. K. Kang, J. M. Kim. Tunneling or value added? Evidence from mergers by Korean business groups. Journal of Finance, 2002, 57(6):2695-2740.

[13] 蔡卫星, 曾诚, 胡志颖. 企业集团、货币 政策与现金持有. 金融研究,2015, 2(416):114-130.

[14] T. Opler, L. Pinkowitz, R. Stulz, R. Williamson. The determinants and implications of corporate cash holdings. Journal of Financial Economics,1999, 52:3-46. 
[15] A. Ozkan, N. Ozkan. Corporate cash holdings: an empirical investigation of UK companies. Journal of Banking and Finance, 2004, 9:2103-2134.

[16] E. I. Altman. Finance ratios discriminant analysis and the prediction of corporate bankruptcy. Journal of Finance, 1968, 23(4):589-609.

[17] D. Martin. Early warning of bank failure: a logit regression approach. Journal of Banking and Finance, 1977, 2:249-276.

[18] J. S. Ohlson. Financial ratio and the probabilistic prediction of bankruptcy. Journal of Accounting Research, 1980, 19:109-131.

[19] M. Lundy. Cluster analysis in credits coring: credit scoring and credit control. Oxford University Press, New York, 1993.

[20] C. F. Huang. Types of credit risks and strategies to improve risk identification by internet of intelligences. Journal of Risk Analysis and Crisis Response, 2013, 3(1): 44-51.

[21] M. Zhang, Z. F. Zhou. A Credit Rating Model for Enterprises Based on Projection Pursuit and K-Means Clustering Algorithm. Journal of Risk Analysis and Crisis Response, 2012, 2(2):131-138.

[22] A. Dittmar, J. Mahrt-Smith, H. Servaes. International corporate governance and corporate cash holdings. Journal of Financial and Quantitative, 2003, 38: 111133. 